\title{
BMJ Open SSRI antidepressant use potentiates weight gain in the context of unhealthy lifestyles: results from a 4-year Australian follow-up study
}

\author{
Zumin Shi, ${ }^{1}$ Evan Atlantis, ${ }^{2}$ Anne W Taylor, ${ }^{1}$ Tiffany K Gill, ${ }^{1}$ Kay Price, ${ }^{3}$ \\ Sarah Appleton, ${ }^{1}$ Ma-Li Wong, ${ }^{4,5}$ Julio Licinio ${ }^{4,5}$
}

To cite: Shi Z, Atlantis E, Taylor AW, et al. SSRI antidepressant use potentiates weight gain in the context of unhealthy lifestyles: results from a 4-year Australian follow-up study. BMJ Open 2017;7:e016224. doi:10.1136/ bmjopen-2017-016224

- Prepublication history and additional material are available. To view these files, please visit the journal online (http://dx.doi. org/10.1136/bmjopen-2017016224).

Received 1 February 2017 Accepted 26 June 2017

\section{(a) CrossMark}

${ }^{1}$ Adelaide Medical School, University of Adelaide, Adelaide, Australia

${ }^{2}$ School of Nursing and Midwifery, University of Western Sydney, Sydney, Australia ${ }^{3}$ School of Nursing and Midwifery, University of South Australia, Adelaide, Australia ${ }^{4}$ Mind \& Brain Theme, South Australian Health and Medical Research Institute, Adelaide, South Australia, Australia ${ }^{5}$ Discipline of Psychiatry, College of Medicine and Public Health, Flinders University, Bedford Park, South Australia, Australia

Correspondence to

Dr Zumin Shi;

zumin.shi@adelaide.edu.au

\section{ABSTRACT}

Objective To examine the association between antidepressant use and weight gain, as well as the interaction with lifestyle factors.

Design Longitudinal study.

Setting and participants We used data from 2334 adults from two stages (4.4 years apart) of the North West Adelaide Health Study, including validated diet and lifestyle questionnaires, measured body weight and linked pharmaceutical prescription data.

Main outcome measures Body weight change.

Results $188(8.1 \%)$ participants had a mean annual number of 1-2 antidepressant prescriptions, and 212 $(9.1 \%)$ had over two prescriptions. The mean annual weight gain was $0.12,0.18$ and $0.28 \mathrm{~kg}$ in non-users, low (1-2 prescriptions/year) and high (>2 prescriptions/ year) antidepressant users, respectively. In multivariable regression models, antidepressant use was positively associated with weight gain: high antidepressant users gained an extra $0.22(95 \% \mathrm{Cl} 0.00$ to 0.44$) \mathrm{kg}$ per year. This association was mainly due to selective serotonin reuptake inhibitor (SSRI) use. High SSRI users gained 0.48 (95\% Cl 0.20 to 0.76$) \mathrm{kg}$ more than non-users. There was no association between tricyclic or other antidepressant use and weight gain. The association between SSRI use and weight gain was stronger among those with high intake of Western diet, greater sedentary activity, and who smoked.

Conclusions SSRIs use was associated with weight gain in the presence of unhealthy behaviours including Western diet, sedentarism and smoking.

\section{INTRODUCTION}

Obesity is a major global health problem almost entirely caused by excess dietary intake and reduced energy expenditure. It is estimated that up to 205 million men and 297 million women over the age of 20 worldwide are obese. ${ }^{1}$ In Australia, the prevalence of obesity class I (body mass index (BMI) $30-34.9 \mathrm{~kg} / \mathrm{m}^{2}$ ) and obesity class II or III (BMI $\geq 35 \mathrm{~kg} / \mathrm{m}^{2}$ ) has respectively doubled and almost tripled since $1980 .^{2}$ Currently it is estimated that $28.3 \%$ of Australian adults

\section{Strengths and limitations of this study}

- Measurement of body weight by health workers at both time points with a mean of 4.4 years of followup;

- Ability to adjust for detailed lifestyle factors and chronic conditions;

- The total number of antidepressant users was relatively small, which limited our power to conduct detailed subgroup analyses;

- Dietary intake was only assessed at follow-up; therefore, we were unable to adjust for dietary change during follow-up.

are obese. ${ }^{3}$ One of the most important health consequences of high and rising trends in global obesity prevalence has been the increased risk of developing depression. ${ }^{4}$ Indeed, data from the Global Burden of Disease study suggest that major depressive disorder was the second leading cause accounting for $8.2 \%$ of global years lived with disability in $2010 .^{5}$

Several population-based cohort studies have consistently shown a positive relationship between antidepressant use and weight gain in countries such as the USA, ${ }^{6-8} \mathrm{Canada}^{9}$ and Australia. ${ }^{10}$ This is valuable information for public health policy-makers and researchers given that the prevalence of antidepressant use is high in Australia and the USA $(5 \%-12 \%),{ }^{711}$ and frequently used by people without depressive or anxiety disorders. ${ }^{12}$

The underlying cause of weight gain due to long-term antidepressant use is poorly understood. ${ }^{13}$ In rodents, data from our laboratory have shown that the combination of chronic stress and short-term antidepressant treatment, followed by high-fat diet, results in long-term weight gain that is greater than that caused by stress and high-fat diet, 
without antidepressant exposure. ${ }^{14}$ In our animal paradigm, antidepressant exposure potentiated weight gain caused by obesity-promoting diet. Thus, increased antidepressant exposure might be a contributory factor to the obesity pandemic. ${ }^{13}$ It is supported by the change of energy intake related to antidepressant use. Data from a recent cross-sectional population-based study showed that antidepressant use was associated with increased energy intake. ${ }^{15}$ Poor diet, sedentary lifestyle, obesity and depression often cluster together; however, association studies between antidepressant use and obesity have been mostly based on registry data or short-term clinical trials, which limit their use to understand interactions. ${ }^{6-10}$ Therefore, whether specific antidepressant medications interaction with lifestyle risk factors (poor diet, inadequate physical activity and smoking) partially explain the development of human obesity is still unclear. Identifying the potential mechanism by which antidepressant medication increases the risk of obesity could help develop targeted strategies for prevention.

This study was designed to specifically examine the association between antidepressant use and weight gain, as well as the interaction with diet and other lifestyle factors (eg, smoking, sedentary activity) in adults participating in a large-population based prospective cohort study.

\section{METHODS}

\section{Data source and study participants}

This study was approved by the Queen Elizabeth Hospital Human Research Committee and, where appropriate, by the Aboriginal Health Research Ethics Committee, Adelaide, South Australia, Australia. The North West Adelaide Health Study (NWAHS) is an ongoing community-based cohort study among adults living in the North West region of Adelaide, South Australia. A detailed description of this cohort has been published elsewhere. ${ }^{16}$ The current study analysed data from both stage 2 (20042006) and stage 3 (2008-2010) data collections. A total of 2334 participants had information on body weight at both time points.

\section{Outcome variable — change in body weight}

At both stages 2 and 3, height and body weight were measured in light clothing and without shoes by trained clinic staff, to the nearest $0.1 \mathrm{~cm}$ and $0.1 \mathrm{~kg}$, respectively. Annual weight gain was calculated by the difference of body weight $(\mathrm{kg})$ between follow-up and baseline divided by the duration of follow-up (in years). Overweight and obesity were defined respectively as $25 \mathrm{~kg} / \mathrm{m}^{2} \geq$ $\mathrm{BMI}<30 \mathrm{~kg} / \mathrm{m}^{2}$ and $\mathrm{BMI} \geq 30 \mathrm{~kg} / \mathrm{m}^{2}$.

\section{Exposure variable-prospective antidepressant use}

Information on medication use (based on prescription) according to the Anatomical Therapeutic Chemical (ATC) Classification was obtained from Medicare Australia (Pharmaceutical Benefits Scheme (PBS)) by confidential unit record linkage for the study period (between baseline and follow-up). Antidepressants (ATC code N06A) were categorised into three groups: tricyclic antidepressants (TCAs) (ATC code N06AA), selective serotonin reuptake inhibitors (SSRIs) (ATC code N06AB) and other antidepressants (ATC code N06AF, N06AG and N06AX). For each participant, the mean annual number of antidepressant prescriptions, calculated by adding the number of prescriptions and dividing it by the follow-up duration between stages 2 and 3, was categorised into three groups: non-user, low user (1-2 prescriptions/year) or high user ( $>2$ prescriptions/year). Exposure of specific antidepressants was assessed independent of one or more antidepressants.

\section{Covariates}

Baseline and follow-up covariates

The Centre for Epidemiologic Studies Depression Scale (CES-D) was used to measure depressive symptoms. CES-D scores were categorised as no depression $(<16)$, mild depression (16-26) or moderate to severe depression $(>26) .{ }^{17}$ Smoking behaviour was determined by self-report and coded as (1) non-smoker and (2) current or ex-smoker. Self-reported income was recoded into three levels $(<\$ A 20$ 000, $\$ A 20000-\$ A 60000$ or $>\$ A 60000)$. Physical activity questions (baseline) from the Australian National Health Surveys were used to classify participants as sedentary, or having low, moderate or high levels of physical activity. ${ }^{18}$ Respondents were asked about the amount of walking, moderate and vigorous activity they had undertaken in the past 2 weeks.

\section{Follow-up only covariates}

Dietary intake during the previous 12 months was assessed by the Cancer Council Victoria Dietary Questionnaire for Epidemiological Studies V3.1 (modification of Food Frequency Questionnaire (FFQ)). The FFQ was previously validated in an Australian population, and is widely used in epidemiological studies. In the analysis, the daily intake of 128 food items were collapsed into 41 food groups as previously described. ${ }^{19}$ Dietary patterns were identified by factor analysis using the principal component method. Varimax rotation was used to assist the interpretability of the factor solution. Based on the Eigenvalue $(>1)$, scree plot and interpretability, two dietary patterns were constructed: (1) the prudent pattern was characterised by high loadings of fruit and vegetable (see online supplementary figure S1) and (2) the Western pattern had high intake of processed meat, snacks and fast food. Scores of each dietary pattern were calculated as the sum of the products of factor loading coefficients and standardised daily intake of the food intake. Dietary pattern scores were dichotomised as low and high (ie, below or above zero).

\section{Statistical analyses}

Analysis of variance and $\chi^{2}$ test were used respectively to compare differences between categorical variables and in continuous variables between groups (gender, categories 
of antidepressant use). Linear regression models were used to assess the longitudinal association between antidepressant use and annual weight change. Three models were employed: model 1 was adjusted for age and gender, model 2 was further adjusted for income, smoking, physical activity and follow-up duration, and model 3 was further adjusted for depression status at baseline and follow-up and dietary patterns (continuous). Participants with missing information of depression were excluded in the corresponding analyses.

As the association between antidepressant use and weight gain was mainly due to SSRI, we further looked at the interaction between SSRI use and lifestyle factors. Multiplicative interaction between SSRI use, lifestyle factors (categorical variables of dietary patterns (low or high), smoking (non-smoker, current or ex-smoker) and physical activity (sedentary, low, moderate/high)) was conducted by inputting the product terms of these variables and antidepressant use in the regression models. The analysis was subsequently stratified for lifestyle factors. The interaction between antidepressant use and age (continuous) was graphically represented using the marginsplot command in STATA V.14 (Stata Corporation).

Sensitivity analyses were conducted using mixed linear modelling to assess the association between antidepressant use and body weight (baseline and follow-up), adjusted for age, income, depression and smoking status as time-varying variables, while considering antidepressant use, physical activity, dietary patterns and gender as time-invariant variables. We also assessed the association (incident rate ratio) between antidepressant use and 5\% weight gain over 5 years using Poisson regression with robust variance.

All analyses were performed using STATA V.14, and statistical significance was set at $\mathrm{p}<0.05$ (two sided).

\section{RESULTS}

The mean age of the sample was 54.1 (SD 14.1) years (table 1). The mean duration of follow-up was 4.4 (SD $0.4)$ years. Women had a higher prevalence of depression and a higher mean level of antidepressant use than men. In the sample, $188(8.1 \%)$ and $212(9.1 \%)$ participants had a mean annual number of $1-2$, and more than 2 antidepressant prescriptions, respectively. Information on antidepressant usage was based on prescription information; out of 400 antidepressant users, 225 (56.3\%) were SSRI users, and in high SSRI users the mean annual number of SSRI prescriptions was 5.9 (SD 3.1) (see online supplementary table $\mathrm{S} 1$ ). The mean annual weight gain was $0.12,0.18$ and $0.28 \mathrm{~kg}$ in non-users, low and high antidepressant users, respectively.

Compared with non-users, high antidepressant users had higher energy intake (9160 vs $8628 \mathrm{~kJ} /$ day) and higher Western dietary pattern scores after adjusting for age and gender (see online supplementary table S2).
In multivariable regression models adjusted for age, gender, income, smoking, physical activity, follow-up duration and dietary patterns, antidepressant use was positively associated with weight gain. High users gained $0.22 \mathrm{~kg}(95 \%$ CI 0.00 to 0.44$)$ per year when compared with non-users, and SSRI use was related to weight gain (table 2). In the fully adjusted model, high SSRI users gained $0.48 \mathrm{~kg}(95 \% \mathrm{CI} 0.20$ to 0.76$)$ more than non-users. No association was found between TCA and other antidepressant use and weight gain.

In relation to annual weight gain, significant interactions were found between SSRI use and three lifestyle factors: Western dietary pattern, smoking and sedentary activity (table 3). The association between SSRI use and weight gain was mainly seen among those with unhealthy lifestyle; a strong dose-response relationship between SSRI use and weight gain was observed among those with high intake of Western diet: the regression coefficients were $0.00 \mathrm{~kg}, 0.46 \mathrm{~kg}$ (95\% CI 0.05 to 0.88 ) and 0.84 $\mathrm{kg}$ (95\% CI 0.43 to 1.24) for non-users, low users and high users, respectively. This association was not seen in those with low intake of Western diet. No significant interaction between SSRI use and the prudent dietary pattern was found. Among those with sedentary lifestyles, high SSRI use was associated with $1.01 \mathrm{~kg}(95 \% \mathrm{CI} 0.52$ to 1.50$)$ higher weight gain per year than non-users. A consistent positive association between SSRI use and weight gain was only observed among smokers: low and high SSRI use was respectively associated with $0.44 \mathrm{~kg}(95 \%$ CI 0.05 to 0.84$)$ and $0.66 \mathrm{~kg}$ ( $95 \%$ CI 0.23 to 1.10$)$ higher weight gain per year than non-users.

There was a significant interaction between antidepressant use and age in relation to weight gain (see online supplementary figure S2). The positive association between high antidepressant use and weight gain was mainly seen among those aged below 50 years.

In the multivariable mixed regression model adjusted for time-varying depression status, smoking, age and income as well as time-invariant dietary patterns, physical activity and gender, antidepressant use was associated with body weight (baseline and follow-up)(see online supplementary table S3). Compared with non-use, high use was associated with an extra body weight of $4.40 \mathrm{~kg}$ (any antidepressant, $\mathrm{p}=0.002$ ), $4.20 \mathrm{~kg}$ (SSRI, $\mathrm{p}=0.007$ ) and $7.14 \mathrm{~kg}$ (other antidepressant, $\mathrm{p}<0.001$ ), respectively. TCA use was not associated with body weight. No interaction between antidepressant use and gender was found (data not shown).

Overall, $27.2 \%$ of the participants had weight gain above $5 \%$ over 5 years. In fully adjusted model, the incident rate ratio (IRR) for $5 \%$ weight gain were $1.00,1.09$ (95\% CI 0.83 to 1.44 ) and 1.37 (95\% CI 1.10 to 1.70$)$ for non-users, low users and high users of antidepressant, respectively. A dose-response association between SSRI use and 5\% weight gain was found in fully adjusted model: IRRs were 1.00, 1.37 (95\% CI 1.03 to 1.81 ) and 1.43 (95\% CI 1.10 to 1.86 ) (p trend $<0.001)$ for non-users, low users and high users of SSRI, respectively (data not shown). 
Table 1 Sample characteristic by sex*



*Values are $\mathrm{n}(\%)$ or mean (SD).

$\dagger$ Annual number of prescriptions.

BMI, body mass index; SSRI, selective serotonin reuptake inhibitor; TCA, tricyclic antidepressant.

\section{DISCUSSION}

In this prospective study, we found that antidepressant use was positively associated with weight gain, which was influenced by significant interactions between SSRI use, age, and unhealthy lifestyle factors, including western dietary pattern, sedentary activity and smoking.

The mean annual weight gain among antidepressant users during this 4.4-year study was around $0.2 \mathrm{~kg}$, which is similar to those reported in the literature for shorter studies. ${ }^{6} 7910{ }^{20}$ However, in those previous population studies, lifestyle factors were either lacking or treated as confounding factors. ${ }^{6} 791020$ None of those studies had been adjusted for dietary intake, an important factor for weight gain.

Only one previous study assessed differences in energy intake and physical activity between antidepressant users and non-users, employing data from the 2005-2006 National Health and Nutrition Examination Survey. It showed that, after adjusting for potential confounding factors, antidepressant users had an extra $215 \mathrm{kcal} /$ day of energy intake and were $77 \%$ more likely to use a computer for $\geq 2$ hour/day than non-users. ${ }^{15}$ The authors 
Table 2 Association $(\beta$ 95\% Cl) between antidepressant use and annual weight gain

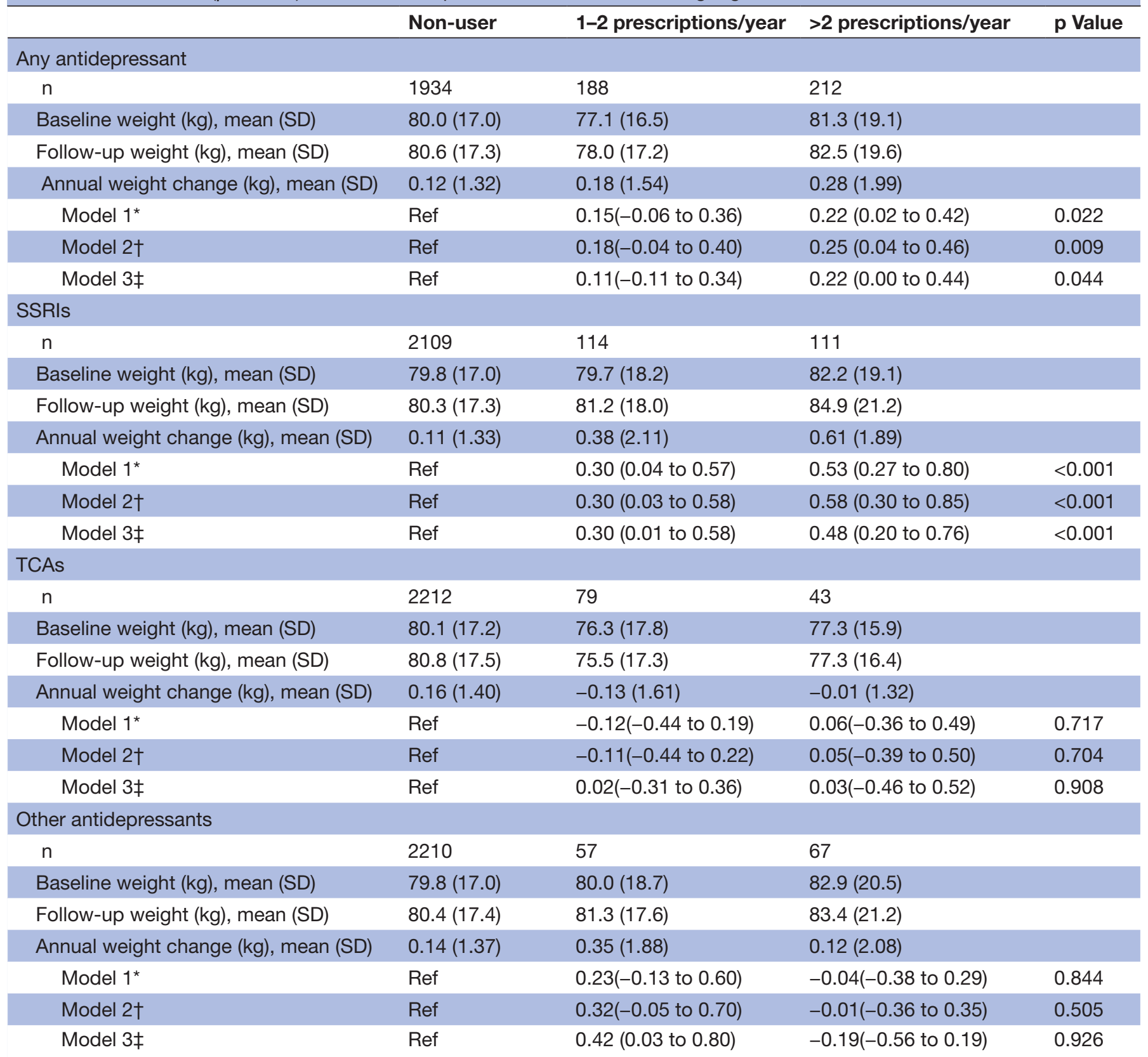

${ }^{*}$ Model 1 adjusted for age and gender.

†Model 2 further adjusted for baseline income, smoking, physical activity, follow-up duration.

$\ddagger$ Model 3 further adjusted for depression status at baseline and follow-up, dietary patterns (continuous).

SSRI, selective serotonin reuptake inhibitor; TCA, tricyclic antidepressant.

hypothesised that increased energy intake and sedentary activity could contribute to weight gain associated with antidepressant use. In the present study, we also found a significant difference in energy intake between high antidepressant users and non-users. After adjusting for age and gender, high antidepressant users had a higher energy intake than non-users (9160 vs $8628 \mathrm{~kJ} /$ day $)$. Furthermore, high antidepressant users had higher Western dietary pattern scores than non-users $(0.14$ vs -0.03$)$. To the best of our knowledge, this is the first study that systematically tested the interactions between antidepressant use and modifiable lifestyle factors. A significant positive dose-response association between antidepressant use and weight gain was found in individuals with high intake but not in those with low intake of Western diet. Clustering of unhealthy behaviours and chronic diseases, including depression, may partly explain the interaction between unhealthy lifestyle and weight gain among those using antidepressant.

The interaction between antidepressant use and smoking in relation to weight gain was consistent with that reported by Arterburn et al who found that 
Table 3 Subgroup analyses of the association between SSRI use and annual weight gain ${ }^{\star}$

\begin{tabular}{|c|c|c|c|c|}
\hline & Non-user & 1-2 prescriptions/year & $>2$ prescriptions/year & $\mathrm{p}$ For interaction \\
\hline Western dietary pattern & & & & 0.026 \\
\hline Low intake & 0.00 & $0.11(-0.29-0.51)$ & $0.14(-0.26-0.54)$ & \\
\hline High intake & 0.00 & $0.46(0.05-0.88) \dagger$ & $0.84(0.43-1.24)$ & \\
\hline Prudent dietary pattern & & & & 0.635 \\
\hline Low intake & 0.00 & $0.35(-0.07-0.78)$ & $0.38(-0.02-0.78)$ & \\
\hline High intake & 0.00 & $0.23(-0.16-0.63)$ & $0.61(0.20-1.02)$ & \\
\hline Physical activity & & & & 0.039 \\
\hline Sedentary & 0.00 & $0.15(-0.34-0.63)$ & $1.01(0.52-1.50)$ & \\
\hline Low & 0.00 & $0.34(-0.13-0.82)$ & $0.23(-0.27-0.72)$ & \\
\hline Moderate/high & 0.00 & $0.33(-0.27-0.94)$ & $0.06(-0.49-0.61)$ & \\
\hline Smoking & & & & 0.002 \\
\hline Non-smoker & 0.00 & $-0.28(-0.73-0.16)$ & $0.35(-0.03-0.72)$ & \\
\hline Current or ex-smoker & 0.00 & $0.44(0.05-0.84)$ & $0.66(0.23-1.10)$ & \\
\hline
\end{tabular}

${ }^{*}$ Bold values represent $p<0.05$.

†Models adjusted for age, gender, income, physical activity, smoking, depression status at baseline and follow-up. Stratifying variables were not adjusted in the corresponding models. Dietary pattern scores are dichotomised as low or high intake. Values represent regression coefficients $(95 \% \mathrm{Cl})$.

SSRI, selective serotonin reuptake inhibitor.

bupropion-treated smokers gained an extra $6.44 \mathrm{~kg}$ compared with fluoxetine-treated non-smokers during a 2-year follow-up study. ${ }^{8}$ We observed an intriguing interaction between antidepressant use and age in relation to weight gain, which may be related to the fact that younger people are more likely to eat a Western diet. In our sample, age was inversely associated with Western dietary pattern scores (data not shown).

The lack of association between TCA use and weight gain was also reported in the Netherlands Study of Depression and Anxiety, as well as the Rotterdam Study. ${ }^{20}{ }^{21}$ However, previous studies have reported an association between TCA use and weight gain. ${ }^{63}$ Our null association between TCA use and weight gain may be due to the fact that age was positively associated with TCA use $(p<0.001)$. The mean age was respectively 53.6, 62.2 and 65.6 years among non-users, low and high users of TCA (data not shown). The SSRI fluoxetine entered medical use in 1986; TCAs were the gold standard for depression treatment before SSRIs became popular. It is likely that older patients started their treatment with TCA and those that were treated with and responded to TCAs for many years before SSRIs became available may have been reluctant to be switched to SSRIs. However, there was no significant age difference between SSRIs users and non-users. Another explanation could be that doctors may be more likely to prescribe SSRIs to people who are worried about weight gain as TCA use has been linked to weight gain in clinical trials.

The strengths of this study include: (1) measurement of body weight by health workers at both time points with a mean of 4.4 years of follow-up; (2) ability to adjust for detailed lifestyle factors and chronic conditions. The main limitation of the study compared with other registry-based studies was that the total number of antidepressant users was relatively small, which limited our power to conduct detailed subgroup analyses. The effect size of the antidepressant use on weight gain may be underestimated due to the fact that some of the low-cost antidepressants (below copayment level) were not recorded by the PBS system before 2012. The PBS dataset only provides information on dispensing not the actual use of antidepressants. Furthermore, dietary intake was only assessed at follow-up; therefore, we were unable to adjust for dietary change during follow-up. There may also be an underestimate or overestimate of energy intake due to the use of FFQ and the inherent issues surrounding recall. Finally, the sample power may be limited for the analyses of TCA and other antidepressants.

Antidepressants are widely used, representing the most prescribed drug class in the $\mathrm{USA}^{22}$; in Australia $11.6 \%$ of the country's population is on antidepressants. ${ }^{23}$ Antidepressant-related weight gain is an outcome of public health relevance, as it may contribute to increased rates of obesity. Here, we provide evidence that antidepressant use was associated with weight gain, especially among those with unhealthy lifestyles, resulting in body weight that is higher than that associated solely with those same lifestyle factors, in the absence of antidepressants. As a matter of public health relevance, SSRI use should be accompanied by proactive efforts to avoid weight gain. We suggest that reducing Western diet consumption, increasing physical activity and smoking cessation may mitigate antidepressant-related weight gain. General practitioners should encourage their patients to adopt healthy lifestyle while 
treating depression with antidepressants or cognitive behavioural therapy.

Contributors ZS contributed to the conception, analysis and interpretation of data; drafting of the report and have given approval of the final version for publication. EA, AWT, TKG, KP, SA, MLW and JL contributed to analysis and interpretation of the data, commented on the report, revising the manuscript and approving the final version for publication.

Competing interests None declared.

Patient consent Obtained.

Ethics approval Queen Elizabeth Hospital Human Research Committee.

Provenance and peer review Not commissioned; externally peer reviewed.

Data sharing statement Data from the North West Adelaide Healthy Study (NWAHS) were accessed from a third party. The authors confirm that for approved reasons, some access restrictions apply to the data underlying the findings. To gain access to the data for this manuscript, ethics approval was sought and granted. Enquiries regarding requests for the NWAHS data can be directed to Prof Robert Adams, Principal Investigator (Clinical) (robert.adams@adelaide.edu.au).

Open Access This is an Open Access article distributed in accordance with the Creative Commons Attribution Non Commercial (CC BY-NC 4.0) license, which permits others to distribute, remix, adapt, build upon this work non-commercially, and license their derivative works on different terms, provided the original work is properly cited and the use is non-commercial. See: http://creativecommons.org/ licenses/by-nc/4.0/

(c) Article author(s) (or their employer(s) unless otherwise stated in the text of the article) 2017. All rights reserved. No commercial use is permitted unless otherwise expressly granted.

\section{REFERENCES}

1. Finucane MM, Stevens GA, Cowan MJ, et al. National, regional, and global trends in body-mass index since 1980: systematic analysis of health examination surveys and epidemiological studies with 960 country-years and 9.1 million participants. Lancet 2011;377:557-67.

2. Atlantis E, Lange K, Wittert GA. Chronic disease trends due to excess body weight in Australia. Obes Rev 2009;10:543-53.

3. Australian Bureau of Statistics. 4364.0.55.001 - Australian Health Survey: FirstResults 2011-12. 2012.

4. Luppino FS, de Wit LM, Bouvy PF, et al. Overweight, obesity, and depression: a systematic review and meta-analysis of longitudinal studies. Arch Gen Psychiatry 2010;67:220-9.

5. Ferrari AJ, Charlson FJ, Norman RE, et al. Burden of depressive disorders by country, sex, age, and year: findings from the global burden of disease study 2010. PLoS Med 2013;10:e1001547.
6. Kivimäki M, Hamer M, Batty GD, et al. Antidepressant medication use, weight gain, and risk of type 2 diabetes: a population-based study. Diabetes Care 2010;33:2611-6.

7. Blumenthal SR, Castro VM, Clements CC, et al. An electronic health records study of long-term weight gain following antidepressant use. JAMA Psychiatry 2014;71:889-96.

8. Arterburn D, Sofer T, Boudreau DM, et al. Long-term weight change after initiating second-generation antidepressants. J Clin Med 2016;5:48.

9. Patten SB, Williams JV, Lavorato $\mathrm{DH}$, et al. Weight gain in relation to major depression and antidepressant medication use. $J$ Affect Disord 2011:134(1-3):288-93.

10. Paige $E$, Korda R, Kemp-Casey A, et al. A record linkage study of antidepressant medication use and weight change in Australian adults. Aust N Z J Psychiatry 2015;49:1029-39.

11. Atlantis E, Sullivan T, Sartorius N, et al. Changes in the prevalence of psychological distress and use of antidepressants or anti-anxiety medications associated with comorbid chronic diseases in the adult Australian population, 2001-2008. Aust N Z J Psychiatry 2012;46:445-56.

12. Harris MG, Burgess PM, Pirkis J, et al. Correlates of antidepressant and anxiolytic, hypnotic or sedative medication use in an Australian community sample. Aust N Z J Psychiatry 2011:45:249-60.

13. Lee SH, Paz-Filho G, Mastronardi C, et al. Is increased antidepressant exposure a contributory factor to the obesity pandemic? Trans/ Psychiatry 2016;6:e759.

14. Mastronardi C, Paz-Filho GJ, Valdez E, et al. Long-term body weight outcomes of antidepressant-environment interactions. Mol Psychiatry 2011;16:265-72.

15. Jensen-Otsu E, Austin GL. Antidepressant use is associated with increased energy Intake and similar levels of physical activity. Nutrients 2015;7:9662-71.

16. Grant JF, Taylor AW, Ruffin RE, et al. Cohort profile: the North West Adelaide Health Study (NWAHS). Int J Epidemiol 2009;38:1479-86.

17. Radolff LS. The CES-D scale: a self-report depression scale for research in the general population. Applied Psychological Measurement 1977;1:385-401.

18. Australian Bureau of Statistics. editor, National Health Survey: Users' Guide. Canberra: ABS, 2003.

19. Schoenaker DA, Dobson AJ, Soedamah-Muthu SS, et al. Factor analysis is more appropriate to identify overall dietary patterns associated with diabetes when compared with treelet transform analysis. J Nutr 2013;143:392-8.

20. Gibson-Smith D, Bot M, Milaneschi Y, et al. Major depressive disorder, antidepressant use, and subsequent 2-year weight change patterns in the Netherlands Study of Depression and Anxiety. J Clin Psychiatry 2016;77:e144-e151.

21. Noordam R, Aarts N, Tiemeier H, et al. Sex-specific association between antidepressant use and body weight in a population-based study in older adults. J Clin Psychiatry 2015;76:e745-e751.

22. IMS Institute for Healthcare Informatics. The use of medicines in the United States: review of 2011, 2012.

23. Mental Health Services in Australia. Mental health-related prescriptions. Secondary mental health-related prescriptions. https:// mhsa.aihw.gov.au/resources/prescriptions/ 